\title{
Orion traducteur : sur C. G. Bjurström
}

Johan Härnsten

\section{OpenEdition}

\section{Journals}

Édition électronique

URL : http://journals.openedition.org/ccs/959

DOI : $10.4000 /$ ccs. 959

ISSN : 2558-782X

\section{Éditeur :}

Presses universitaires de Rennes, Association des lecteurs de Claude Simon

\section{Édition imprimée}

Date de publication : 30 avril 2015

Pagination : 157-166

ISBN : 9782753539990

ISSN : 1774-9425

Référence électronique

Johan Härnsten, «Orion traducteur : sur C. G. Bjurström », Cahiers Claude Simon [En ligne], 10 | 2015, mis en ligne le 22 septembre 2017, consulté le 01 mai 2019. URL : http://journals.openedition.org/ ccs/959; DOI : 10.4000/ccs.959 


\title{
ORION TRADUCTEUR: SUR C. G. BJURSTRÖM
}

\author{
Johan HÄRNSTEN \\ ENS Ulm et Université Paris 8
}

Le nom de Carl Gustaf Bjurström (1919-2001) ne peut manquer de susciter un sentiment d'admiration et de dette. Traducteur de Céline, Camus et Michaux, installé dès 1948 à Paris où il fut directeur de l'Institut suédois dans les années 1950, Bjurström est simplement le traducteur suédois de littérature française du $\mathrm{Xx}^{\mathrm{e}}$ siècle. Connu en Suède surtout pour ses traductions des auteurs du Nouveau Roman - Nathalie Sarraute, Michel Butor, et avant tout Claude Simon -, il fit également connaître au public français des auteurs suédois comme August Strindberg, Stig Dagerman et Gunnar Ekelöf, dans un grand nombre de traductions et d'articles. Comme le résume succinctement Mathieu Lindon dans son hommage au traducteur, " [s]ans Carl Gustaf Bjurström, [...] les littératures scandinaves en France ne seraient pas ce qu'elles sont, de même que la littérature française en Scandinavie ${ }^{1}$ ".

Cet article vise en premier lieu à donner un aperçu de l'œuvre de Bjurström: de ses traductions, vers le français et vers le suédois. Il s'agira ensuite de regarder de plus près ses traductions de Claude Simon, seul auteur français que Bjurström a traduit de manière systématique, avec le succès qu’on sait $^{2}$. Nous nous appuierons sur l'importante correspondance menée entre Simon et Bjurström, laquelle fait partie de l'archive que le traducteur a confiée à la Bibliothèque royale ${ }^{3}$ de Stockholm (Kungliga biblioteket) peu avant son

1. M. Lindon, « Mort d'un passeur suédois », Libération, le 31 août 2001.

2. Sur l'attribution du prix Nobel en 1985 et la réception de l'œuvre simonienne dans les pays nordiques (Suède, Danemark, Norvège), voir l'étude très détaillée de Karin Holter, "Voici vingt-cinq ans le prix Nobel. La réception de Claude Simon en Scandinavie ", dans Cabiers Claude Simon, n 6, 2010, Perpignan, Presses Universitaires de Perpignan, 2010, p. 135-162.

3. Je tiens à remercier Anne Scherman et Jan-Eric Ericson de la Bibliothèque royale de Stockholm pour leur assistance. Les lettres citées dans cet article viennent toutes de l'archive de Bjurström (Kungliga biblioteket, réf. HS L 281). Un merci spécial à Inger Bjurström. 
décès. Quels problèmes spécifiques l'écriture simonienne pose-t-elle à la langue suédoise? À partir des questions ouvertes par la correspondance et par certains écrits du traducteur lui-même, il s'agira enfin de voir ce que Bjurström a dit plus généralement sur la pratique de la traduction.

\section{LA BIBLIOGRAPHIE DE BJURSTRÖM}

L'impressionnante bibliographie ${ }^{4}$ de Bjurström comprend plus de 900 publications: 158 traductions en suédois, 253 traductions vers le français, ainsi que quelque 500 textes - en français ou en suédois - en son propre nom, notamment une série de "Lettres de Paris" " (Brev från Paris) qui, dès la fin des années 1940, présentaient au public suédois des auteurs tels que Raymond Queneau et Maurice Blanchot. Auteur d'un grand nombre d'articles publiés dans le quotidien suédois Dagens Nyheter, dont il fut le correspondant régulier pendant plusieurs décennies, il publiait aussi régulièrement en français - on lui doit par exemple une contribution sur les littératures scandinaves dans Le Grand Larousse encyclopédique.

Moins connues en Suède sont ses très nombreuses traductions françaises d'écrivains suédois, toujours réalisées en collaboration avec un traducteur ou auteur français. Nous ne pouvons évoquer ici que quelques pans de ce travail, très important: il faut mentionner d'abord la traduction d'August Strindberg, dont Bjurström a traduit et édité l'Euvre autobiographique, parue au Mercure de France, et le Théâtre complet, publié en six tomes à l'Arche. Commencé en collaboration avec Boris Vian ${ }^{6}$, et poursuivi principalement avec Georges Perros $^{7}$, ce travail monumental l'a occupé des années 1950 jusqu'à la fin de sa vie. Avec André Mathieu, ensuite, Bjurström a traduit la trilogie Diwan du poète suédois Gunnar Ekelöf, travail auquel il attachait également une grande importance ${ }^{8}$. Aux côtés de Lucie Albertini, enfin, il a traduit plusieurs ouvrages de Stig

4. Voir l'indispensable outil établi par Bodil Gustavsson (éd), C.G. Bjurström. En bibliografi, Acta Bibliothecæ regiæ Stockholmiensis LXI, Stockholm, Kungliga biblioteket, 1999.

5. Publiées dans la revue Bonniers litterära magasin (BLM).

6. Sur cette collaboration, voir C. G. Bjurström, "Boris Vian, traducteur de Strindberg ", Obliques, n 8-9, 1976, p. 291-294.

7. Les Lettres à Carl Gustaf Bjurström (1958-1976) de Georges Perros sont éditées chez La Part Commune (1999).

8. Dans une lettre du 18 octobre 1985, le lendemain de l'annonce du prix Nobel, Bjurström écrit à Claude Simon que la publication de cette trilogie d'Ekelöf faisait partie, avec la parution en langue française du théâtre de Strindberg, des " trois choses " qu'il voulait voir se réaliser avant de disparaître. Il conclut: "Celle qui ne dépendait pas de moi était votre prix Nobel.» 
Dagerman, édités chez Denoël; des textes d'Ingmar Bergman et de Lars Norén, ainsi qu'un grand nombre de poètes danois et norvégiens.

Vers le suédois, Bjurström a commencé par traduire Julien Gracq (Vid Syrtens stränder $)^{9}$ et Henri Michaux (En barbar i Asien), pour continuer avec Albert Camus, dont il a traduit plusieurs livres, et auquel il a consacré une monographie en 1957. Bjurström a aussi été un des premiers à introduire Le Clézio en Suède (La Fièvre et Le Déluge, traduits en 1966 et en 1968). On lui doit également la traduction de nombreux textes théoriques et philosophiques, parmi lesquels L'Archéologie du savoir et Surveiller et punir de Michel Foucault. Sa traduction du Voyage au bout de la nuit, publiée en 1971, a fait date en Suède: marquée par une langue "fautive " qui retranscrit l'oralité du suédois, cette traduction a été très lue et aimée (critiquée aussi) et semble avoir marqué une certaine génération d'auteurs qui se sont inspirés de son style.

Toutefois, pour le public suédois, Bjurström est surtout connu comme le traducteur des auteurs du Nouveau Roman: Nathalie Sarraute (Enfance), Michel Butor, Robert Pinget, Alain Robbe-Grillet et Samuel Beckett. Dans cet ensemble, Claude Simon occupe une place privilégiée: du Vent jusqu'au Jardin des Plantes, c'est le seul auteur dont Bjurström a traduit la quasi-totalité de l'œuvre: 13 romans ainsi que quatre textes courts.

\section{LA CORRESPONDANCE SIMON-BJURSTRÖM}

La correspondance entre Simon et Bjurström, comprenant quelque 160 lettres, débute en 1961, lors de la publication suédoise du Vent, et se termine peu avant le décès du traducteur, en 2001. Les lettres à Simon - des tapuscrits, dont l'archive garde les copies carbone - témoigne de l'extrême dévouement de Bjurström à son travail de traducteur. Il fait régulièrement le point sur la réception de l'œuvre simonienne, avec des envois fréquents de critiques qui viennent de paraître dans la presse suédoise, traduites uniquement pour Simon. Parallèlement à la traduction proprement dite, il y a un véritable travail d'accompagnement de l'œuvre traduite, grâce auquel Simon est tenu au courant de l'édition étrangère et des différentes réactions à ses romans. Bjurström fait également parvenir ses propres articles sur

9. Pour une belle analyse de cette traduction, voir Vincent Fournier, «Julien Gracq et Marcel Proust à l'épreuve de la traduction suédoise" dans Deshima, hors-série nº 3 (dir. S. Briens et M. Kylhammar): "Le Nord à la lumière du Sud. Mélanges offerts à Jean-François Battail ", Strasbourg, Université de Strasbourg, 2013, p. 221-227. 
l'auteur - il est l'auteur d'une dizaine de textes sur Simon ${ }^{10}$, et il annonce à plusieurs reprises au début des années 1980 son intention de les réunir dans un livre. Cet ouvrage n'a jamais vu le jour. Simon reçoit aussi des livres que Bjurström a traduits en français, et les lettres de l'auteur (toujours manuscrites) font entendre un fin lecteur d'écrivains suédois ${ }^{11}$.

La correspondance montre surtout que le travail de traduction s'élabore dans un dialogue continu entre auteur et traducteur. À titre d'exemple, on peut mentionner la longue lettre du 21 mars 1964, une lettre de 15 pages, dont 7 sont des traductions de critiques suédoises de L'Herbe, ouvrage paru en Suède en 1963. Dans cette lettre, Bjurström sollicite des clarifications sur des passages du Palace qu'il trouve obscurs (concernant par exemple la description des fenêtres de l'hôtel) et demande à Simon d'expliquer l'ordonnancement des parenthèses. Il soulève le problème des jeux de mots polyglottes qui figurent dans le roman - celui, notamment, de l'Américain qui associe "assaisonner " et l'espagnol asesinado. Dans la mesure où un équivalent hispano-suédois n’en est pas possible («ces langues sont trop loin l'une de l'autre »), Bjurström propose une traduction qui déplace considérablement le sens de la phrase, solution que Simon refusera dans sa réponse, suggérant à la place l'introduction d'une note en bas de page explicative.

Bjurström relève aussi des fautes dans les quelques phrases en espagnol qui figurent dans l'original, où l'on lit par exemple ¿Quién a muerto? au lieu de ¿Quién ha muerto? Simon valide presque toutes les corrections proposées par le traducteur, et la plupart des modifications seront intégrées dans la réimpression française du roman en 1991. Faut-il traduire l'original jusque dans ses incorrections? Dans sa communication à Arles en 1985, Bjurström évoque un exemple similaire: Simon parle dans La Bataille de Pharsale d'un "avion qui trace sur le ciel le mot ORION et nous lisons que "l'avion publicitaire entame le dernier jambage de la sixième lettre N” ». Or, le mot Orion ne comporte que cinq lettres.

10. Pour n'en citer que trois: "Dimensions du temps chez Claude Simon", Entretiens, Rodez, 1972 (31), p. 141-158; "Lecture de Claude Simon: Le Vent », Critique, 1981 (414), p. 1151-1161; " Att läsa Claude Simon: dialog och spegelbilder i Gräset ", Artes, 1986 (12: 1), p. 3-13.

11. Simon admire les Ennuis de noce de Dagerman - « on y sent un souffle poétique qui vient équilibrer (ou fautil dire compléter?) la force rabelaisienne par une tendresse et une émotion rares " - mais se montre sceptique à l'égard de Bergman: « Merci infiniment [...] de votre remarquable traduction de De la vie des marionnettes que j'ai lue avec grand plaisir, quoique je doive vous avouer que le psychisme des personnages de Bergman me soit bien étranger ». Lettres de Simon à Bjurström du 30 mars 1982 et du 7 novembre 1980. 
Cela appartient aussi au travail du traducteur: relever les inconséquences éventuelles du texte et demander à l'auteur si elles sont voulues - auquel cas il faut bien entendu les respecter -, ou si elles sont involontaires et s'il permet alors au traducteur de faire une petite correction ${ }^{12}$.

Si la correspondance entre Simon et son traducteur suédois montre un auteur qui intervient parfois dans la traduction de ses livres, l'inverse est aussi vrai : la traduction implique un retour sur le texte de départ qui ne peut pas toujours laisser celui-ci indemne. Le processus de traduction s'avère un travail de clarification, de correction - et même, pourrait-on dire, de réécriture - du texte source lui-même, visant à établir la traduisibilité de celui-ci. La traduction, en somme, représente aussi l'élaboration d'un original qui, en toute rigueur, n'existait pas avant l'acte de traduction.

\section{QUE « TRADUIT » LE PARTICIPE PRÉSENT ?}

À part ces questions de détail, Bjurström évoque aussi dans la correspondance des problèmes plus généraux liés à la traduction de la phrase simonienne. Il est question, notamment, du participe présent, abondamment utilisé par Simon, et qui représente, à en croire les témoignages de la table ronde consacrée à Simon à Arles en 1985, un problème communément partagé par ses traducteurs. Comme l'explique Bjurström dans son intervention,

[1]e participe présent - que Claude Simon utilise de façon déjà fort inusitée en France - arrive difficilement à obtenir le même statut en suédois. En français il se termine par une syllabe, " ant ", qui s'évanouit de façon somme toute assez harmonieuse. En suédois il se termine par deux syllabes, "ande ", ce qui fait beaucoup plus monotone, si on l'utilise à longueur de page, et qui en plus me semble légèrement boiteux: "klappande, knakande, tittande " ${ }^{3}$.

Toutefois, la question n'est peut-être pas tant de savoir comment traduire ce mode du verbe que d'identifier ce qu'il est lui-même censé produire comme effet - ce qu'il est lui-même censé " traduire ", en quelque sorte. Claude Simon s'est souvent prononcé sur son usage du participe présent, et il est frappant de constater qu'il a recours (il est vrai métaphoriquement) au terme de traduction:

J'étais hanté par deux choses: la discontinuité, l'aspect fragmentaire des émotions que l'on éprouve et qui ne sont jamais reliées les unes aux autres, et en même temps leur contiguïté dans la conscience. Ma phrase cherche à traduire cette contiguïté. L'emploi du participe présent me permet de me placer hors du temps conventionnel. Lorsqu'on dit: il alla à tel endroit, on donne

12. Table ronde sur "Claude Simon et ses traducteurs européens ", Actes des deuxièmes assises de la traduction littéraire (Arles 1985), Arles, Actes Sud, 1986, p. 100-101.

13. Ibid., p. 97-98. 
l'impression d'une action qui a un commencement et une fin. Or, il n'y a ni commencement ni fin dans le souvenir ${ }^{14} \ldots$

Ce qu'il faut traduire, c'est précisément cet effet de simultanéité des événements, si caractéristique de l'espace-temps simonien - un mouvement figé, immobile, où les actions sortent de la hiérarchie chronologique pour se côtoyer dans ce grand présent, "sans début ni fin ", qu’est le temps singulier du souvenir. Pour Simon, cette présence est corrélativement celle de l'écriture même. Si le participe présent fait tendre le narratif vers le descriptif, le verbal vers l'adjectival, il a aussi pour effet d' "injecte[r] du tempsdans [1] a description ${ }^{15}$ ", selon la formule de David Zemmour: les descriptions se voient dotées d'une temporalité interne, une temporalité que Bjurström décrit comme "intensive », voire "explosive ${ }^{16}$ ". Le traducteur suédois peut ainsi conclure son article dans le recueil Orion Blinded en affirmant que Simon a réussi à faire "sourdre " du temps du texte lui-même - Claude Simon... knows how to make time well up from the text itself $f^{7}$.

C'est donc cette étrange temporalité du souvenir, telle qu'elle se présente à la conscience, qu'il faut traduire. Mais comment la rendre dans une langue qui, comme le suédois, se sert très peu du participe présent? Pour sauver l'effet de simultanéité des actions, Bjurström souligne qu’il lui a fallu

trouver des tournures qui restituent par d'autres moyens cette expérience réduite à une qualité. Ces tournures sont multiples et tout dépend du verbe et de sa place dans la phrase, mais je me suis souvent servi de la tournure "et lui qui faisait ceci ou cela... " 18

En réalité, Bjurström a recours à plusieurs tournures: des propositions relatives ou circonstancielles, parfois introduites par des conjonctions comme " tandis que ", "en même temps que "; souvent un simple och (" et »), conjonction qui connaît un usage assez souple en suédois et qui permet à la fois de scander et de relancer la phrase, un peu à la manière dont la virgule sépare les propositions

14. Entretien avec Claude Sarraute dans Le Monde, 8 octobre 1960, cité dans D. Zemmour, "Le participe présent dans La Route des Flandres: Écriture du souvenir et quête de l'instant" dans L'Information grammaticale, $\mathrm{n}^{\circ} 76,1998$, p. 42.

15. Ibid., p. 44 .

16. Voir "Claude Simon et ses traducteurs européens ", op. cit., p. 98: "Ce qui intéresse dans le participe présent [...] de Claude Simon, c'est que l'action y est concentrée, gelée, explosive en quelque sorte ».

17. C. G. Bjurström, «Composition, Repetition, and Dislocation in Simon's First Three Novels », trad. Jane Carson, dans Orion Blinded. Essays on Claude Simon, dir. R. Birn et K. Gould, Lewisburg, Bucknell University Press, 1981, p. 172.

18. "Claude Simon et ses traducteurs européens ", op. cit., p. 98. 
participiales simoniennes ${ }^{19}$. Si l'effet de simultanéité est ainsi sauvé, c'est bien sûr le côté systématique, répétitif et insistant du participe simonien qui a tendance à s'estomper.

Quand il n'est pas rendu par un adjectif ou par un indicatif présent, comme c'est le cas dans certaines descriptions "pures ", le participe présent est le plus souvent traduit par un prétérit suédois, temps par excellence de la narration, ainsi que le montrent ces lignes de La Route des Flandres:

puis le tout - homme cheval et sabre - s'écroulant d'une pièce sur le côté comme un cavalier de plomb commençant à fondre par les pieds et s'inclinant lentement d'abord puis de plus en plus vite sur le flanc, disparaissant le sabre toujours tenu à bout le bras derrière la carcasse de ce camion brûlé effondré là ${ }^{20} \ldots$

och så störtade alltihopa - häst ryttare och sabel - i ett enda stycke ner på sidan, som en tennsoldat som börjar smälta nere vid fötterna och som först sakta och sedan allt fortare lägger sig ner på sidan, och så försvann han med sabeln alltjämt i högsta hugg bakom skrovet av en brinnande lastbil som sjunkit ihop där ${ }^{21} \ldots$

Comme on pouvait s'y attendre, c'est précisément le côté « hésitant » du participe qui disparaît dans une telle traduction: il devient tantôt adjectif, tantôt, comme dans le passage cité, verbe "véritable ». Le prétérit suédois confère aux actions un caractère accompli - le « disparaissant » devient une vraie disparition (försvann, "disparut»), désormais attribuée au pronom "il »(han), qui régit la dernière proposition suédoise (" et puis il disparut...»), tandis que dans le texte français, le participe fonctionne sans support, faisant disparaître le sujet même de la disparition. En revanche, le suédois transforme un participe passé en participe présent: le camion, " brûlé » chez Simon, est toujours " en flammes " (brinnande) chez Bjurström. L'introduction de propositions subordonnées entraîne des problèmes d'un autre genre: les pronoms relatifs "qui » et "que » se traduisent en suédois par le même mot que "comme", ce qui crée rapidement une accumulation du mot som dans le texte suédois (som en tennsoldat som börjar smälta: " comme un cavalier de plomb qui commence à fondre ") - et qui produit parfois des confusions ${ }^{22}$.

19. À Arles en 1985, Bjurström évoque ainsi « le problème que posent [les] séries de mots et de phrases qui se succèdent, sans conjonctions de coordination et uniquement séparés par une virgule - qui ne se prononce pas, mais qui correspond à une respiration. En suédois [...] on a tendance à introduire de temps en temps un "et" ". Ibid., p. 97.

20. C. Simon, La Route des Flandres, Minuit, coll. « Double ", 1960, p. 13.

21. C. Simon, Vägen till Flandern, trad. C. G. Bjurström, Stockholm, Gebers, 1962, p. 9-10.

22. "Je me rappelle m'être un jour plaint à Claude Simon de cette confusion, mais contrairement à mon attente il m’a félicité de pouvoir ainsi écrire en suédois le mot "som" aussi bien pour "comme" que pour "qui" ". C. G. Bjurström, "Claude Simon et ses traducteurs européens ", op. cit., p. 98. 


\section{«LA MAIN ET L'OREILLE »: TRADUIRE LA DÉMARCHE DE L'AUTEUR}

Comment traduire? Sans jamais établir de véritable "méthode " de la traduction, Bjurström a souvent insisté sur le fait que le traducteur doit avoir une certaine liberté et qu'il doit pouvoir se permettre de se fier à l'intuition. Le traducteur n'a pas nécessairement à respecter la stricte grammaticalité de l'original, ni à essayer de saisir l'intention derrière l'œuvre - c'est d'ailleurs ce que Simon n'a cessé de répéter: le sens ne préexiste pas à l'écriture; il en procède plutôt. Il arrive à Bjurström de revendiquer une approche " manuelle ", tactile, de la langue, approche qu'il oppose à une conception trop intellectuelle. La langue, avant d'être un ensemble de signes, est d'abord une matière qu'il revient au traducteur de saisir, de manier et de maîtriser, presque manuellement. Cette matière-langue, Bjurström l'a parfois comparée au bois. Dans une table ronde consacrée à l'œuvre de Julien Gracq, Bjurström développe cette métaphore menuisière:

La prose de Claude Simon est, pour moi, d'un bois souple qui se laisse couper en longs copeaux, tandis que celle de Michel Butor me fait penser à un bois de fibres courtes qui se casse et qui fait des échardes qui s'enfoncent sous les ongles. [...] [A]yant cette sensation manuelle de la traduction, il m'est difficile d'analyser en lisant, il faut que j'y mette la main [...]. [L]a main et l'oreille [...] sont plus précieuses que l'intellect ${ }^{23}$.

Dans un article intitulé « Le travail du traducteur ", Bjurström explique aussi que c'est à partir de cette saisie manuelle de la langue, seulement, que l'original peut, lui, être pleinement "saisi » ou compris - il joue ici sur l'affinité des mots gripa et begripa, saisir et comprendre ${ }^{24}$. C'est dans la langue cible, seulement, que le traducteur « comprend » ou "se saisit de » l'œuvre à traduire.

Ce que doit faire le traducteur, ce n'est pas «faire passer " une œuvre d'une langue à une autre - implicitement et de manière étonnante, Bjurström remet en cause l'idée communément acceptée de la traduction comme passage, trop abstraite selon lui dans la mesure où elle implique que l'œuvre serait en quelque sorte séparable des deux langues, ou qu'il y aurait quelque chose " entre " les langues que précisément on pourrait faire "passer ». Qu'est-ce que la traduction, si elle n'est pas passage? Le traducteur, dit Bjurström, peut adopter la démarche de l'auteur (författarens tillvägagångssätt); il peut faire sienne l'attitude qui est celle de l'écrivain vis-à-vis de la langue. Dans la table ronde à Arles consacrée à Claude Simon, Bjurström explique que

23. "Julien Gracq et ses traducteurs. Traduction du style et style de la traduction ", Actes des onzièmes assises de la traduction littéraire (Arles 1994), Arles, Actes Sud, 1995, p. 107.

24. C. G. Bjurström, «Översättarens arbete » dans Svensk litteraturtidskrift, nº 2, 1978 (41), p. 13-17. 
[c]e n'est pas par une analyse intellectuelle préliminaire que j'arrive à comprendre ce qu'un texte a dans le ventre, mais en écrivant les mots, en tâchant d'équilibrer les phrases et en m'efforçant de reproduire non pas tant le texte lui-même que le travail de l'auteur, tel qu'il s'est déroulé dans sa langue ${ }^{25}$.

Ce qu'il faut traduire, ce n'est pas le français, ni, en un certain sens, la phrase simonienne, mais l'impact de cette phrase dans la langue. Ce qu'il faut traduire, c'est l'événement Claude Simon dans la langue française - il faut traduire l'écriture, pour employer un terme cher à Simon, ce qui ne veut donc pas dire la calquer.

Or, cette stratégie - traduire la démarche de l'auteur - suppose nécessairement une certaine liberté du traducteur, liberté sur laquelle Bjurström s'explique aussi. Elle signifie d'abord que le traducteur n'a pas toujours à être fidèle à la lettre du texte original, aux particularités locales de l'œuvre qu'il traduit: ce qu'il faut produire, c'est un tout, une entité organique. L'unité à traduire n'est ni le mot, ni la phrase, mais le roman dans son ensemble: c'est à ce niveau, seulement, qu'on peut parvenir à produire quelque chose comme une "équivalence". Les mots et les phrases ne prennent sens que les uns par rapport aux autres: c'est toujours «l'ensemble qui compte, et les rapports de la partie avec cet ensemble ${ }^{26}$ ». Bjurström souligne l'inanité de l'équivalence locale et va même jusqu'à dire que "des phrases isolées ne sont pas traduisibles ${ }^{27}$ ".

Cette vision d'ensemble de l'œuvre rend possible ce que Bjurström appelle l'équivalence globale ou décalée - c'est-à-dire, en somme, que des passages intraduisibles peuvent être "rattrapés " à un autre endroit du texte, lorsque la langue en fournit l'occasion - un peu comme l'effet du participe présent peut être rendu par d'autres moyens.

Il y a des jeux de mots qui font pour ainsi dire fonction de charnières dans un récit et auxquels il faut absolument trouver une équivalence à l'endroit même où ils se trouvent. Il y en a qui dénotent simplement une certaine attitude en face du langage et de ses possibilités et qui ne doivent inciter le traducteur qu'à chercher à prendre lui-même la même attitude, sans essayer de reproduire à chaque instant et au moment même, le jeu de mots de l'original. Quand Queneau parle d'un « chevable vénérable » il est pratiquement impossible de trouver dans chaque langue une traduction exacte, exactement à cet endroit - mais ce n'est pas ce que nous demande le texte de Queneau qui nous incite seulement à faire une pirouette du même genre quand les mots de la langue cible s'y prêtent ${ }^{28}$.

25. "Claude Simon et ses traducteurs européens ", op. cit., p. 95.

26. Ibid., p. 96.

27. Lösryckta meningar kan inte översättas. " Översättarens arbete », art. cit., p. 15.

28. "Claude Simon et ses traducteurs européens ", op. cit., p. 96. Nous soulignons. 
Proche ici de la conception de la traduction défendue par Henri Meschonnic, prônant une attention au " faire " de l'auteur devant le " dire " explicite du texte, Bjurström aurait pu souscrire à cette phrase de Poétique du traduire: la traduction doit " être une invention du discours, si ce qu'elle traduit l'a été ${ }^{29}$ ".

\section{CARREFOURS DE SENS}

Si fidélité il devait encore y avoir, dans la traduction, elle est à penser non pas à l'égard de la lettre de l'original, mais plutôt envers cette " attitude face aux possibilités de la langue ": envers le travail de l'écrivain, sa "démarche " dans la langue. Le traducteur, enfin, doit être à l'écoute des potentialités de sa propre langue, en tant qu'elle offre à chaque instant des solutions imprévues, des possibilités multiples. Comment ne pas voir, alors, que Bjurström retrouve une idée que Simon avait développée dans sa préface à Orion aveugle et qu'il rappelle à Stockholm en 1985, lors du prix Nobel? "Les mots [...] ne sont pas seulement des "signes" mais [...] carrefours de sens, de sorte que déjà par son seul vocabulaire la langue offre la possibilité de "combinaisons" en "nombre incalculable" ${ }^{30}$. " Il nous semble que Bjurström, dans son idée de l'équivalence décalée, et dans la définition qu'il donne de la traduction comme activité tactile, manuelle, nécessitant avant tout "la main et l'oreille", s'approche de l'idée simonienne d'un cheminement tâtonnant dans la langue, allant de "carrefour" en "carrefour". Semblable en cela non pas tant à un Hermès messager, mais plutôt à cet Orion aveugle décrit par Simon, le traducteur doit, lui aussi, parfois fermer les yeux pour mieux entendre ce que la langue lui souffle comme possibilité.

Les «carrefours du texte ${ }^{31}$ " simonien ne sont-ils pas aussi un ensemble de bifurcations en langues différentes? Les chemins de l'œuvre - historiques et géographiques - ne sont-ils précisément pas ce que les traductions permettent exemplairement d'étudier? Force est de dire que les traductions des romans de Simon constituent autant de " carrefours " qui, loin de fixer le sens de son œuvre, contribuent au contraire à en rendre le cheminement toujours plus imprévisible.

29. H. Meschonnic, Poétique du traduire, Verdier, 1999, p. 460. Nous soulignons.

30. C. Simon, Discours de Stockholm, Minuit, 1986, p. 28. Nous soulignons.

31. Voir l'ouvrage de R. Sarkonak, Claude Simon: Les Carrefours du texte, Toronto, Paratexte, 1986. 